\title{
Was können wir von Ablehnung lernen? Eine Befragung von NichtnutzerInnen im Kontext einer Produktentwicklung
}

Laura Augustin, Sabrina Pfrang, Björn Kokoschko, Andrea Wolffram, Michael Schabacker

Die Humanzentrierung stellt den Menschen in den Mittelpunkt der (Weiter-)Entwicklung von (neuen) Produkten. Der Fokus liegt jedoch hierbei hauptsächlich auf den Menschen, die das betreffende Produkt besitzen oder nutzen. Nichtnutzung und Ablehnung wird im Kontext der NutzerInnenfreundlichkeit allerdings seltener betrachtet, kann jedoch wertvollen Input für eine Produktentwicklung bieten, indem mehr als nur das Negativfeedback von Nutzenden betrachtet wird. Dieser Beitrag befasst sich mit dem Thema Nichtnutzung im Kontext der Produktentwicklung und wie der Aspekt Ablehnung zukünftig eingebunden werden kann. Im Rahmen einer Fallstudie werden NutzerInnen und NichtnutzerInnen von Kaffeeautomaten über ihre Nutzung bzw. Ablehnung des Produkts befragt, um die Ergebnisse anschließend für eine umfassende Produktentwicklung aufzubereiten. Es wird untersucht inwiefern sich die Ergebnisse unterscheiden und welche davon für eine Produktentwicklung wertvoller sind. Es werden Vorschläge gemacht, wie die Ergebnisse aufbereitet werden können, zum Beispiel mithilfe der sogenannten Non-Persona, die das Konzept der Persona auf Nichtnutzung übersetzt. Abschließend werden Empfehlungen für die zukünftige Einbindung von NichtnutzerInnen in der Produktentwicklung gegeben.

Keywords: NichtnutzerIn, NutzerInnenzentrierung, Produktentwicklung, Nutzerbefragung, User Experience

\section{Einleitung}

Mit der wachsenden Popularität der Humanzentrierung und Integration von NutzerInnenfeedback in der Produktentwicklung (Gardan, 2017; Kurosu, 2011) erhalten NutzerInnen immer größeren Stellenwert bei der Entwicklung von Produkten und Services. Mit den Methoden wie unter anderem User Journeys aus dem Marketing (Lemon \& Verhoef, 2016), der Journey Map (Doorley, Holcomb, Klebahn, Segovia \& Utley, 2018) oder Personas (Nielsen, Hansen, Stage \& Billestrup, 2015) werden Wünsche und Kritiken gesammelt und visuell aufbereitet. Mit Vorgehen wie dem Design Thinking (Brenner, Uebernickel \& Abrell, 2016) oder Scrum (Sutherland \& Schwaber, 1993) werden NutzerInnen in jeder Phase der Produktentwicklung miteinbezogen, sei es nun in der 
Ideenfindung oder durch regelmäßiges Feedback zu ersten Konzepten. Methoden, die hier Anwendung finden, sind zum Beispiel integrativer Art wie die Entwicklung zusammen mit verschiedenen Stakeholdern mithilfe der Co-Creation (Ind \& Coates, 2013). Der positive Effekt der Einbindung von Nutzung in die Produktentwicklung ist unumstritten (R. G. Cooper \& Kleinschmidt, 1986; Hippel, 1976; Murphy \& Kumar, 1997). NichtnutzerInnen sind umfassend definiert (Gardan, 2017; Kurosu, 2011), Nichtnutzung ist jedoch noch nicht abschließend betrachtet worden (Augustin, Kokoschko, Wiesner, Schabacker, 2020; Barsch, Heupel \& Trautmann, 2019; Ribak \& Rosenthal, 2015; Satchell \& Dourish, 2009; Wyatt, 2003). Unterschiedliche Disziplinen haben sich bisher mit der Definition der Nichtnutzung befasst (Satchell \& Dourish, 2009; Wyatt, 2003), jedoch noch nicht mit der Einbindung der NichtnutzerInnen in einem Entwicklungsprojekt. Dieser Beitrag untersucht aufbauend auf der Definition von Nichtnutzungstypen (Augustin, Kokoschko, Wiesner, Schabacker, 2020) und Gründen für eine Nichtnutzung (Augustin, Kokoschko, Wolffram, Schabacker, 2021) im Rahmen einer Produktentwicklung die beiden Aspekte Nutzung und Nichtnutzung. Hierbei wird genauer beleuchtet, weshalb NutzerInnen ein bestimmtes Produkt nutzen und wieso die Nichtnutzerlnnen dieses ablehnen. Dies birgt das große Potential eine vollkommen neue NutzerInnengruppe zu erschließen und das Produkt oder den Service auch für bisherige NutzerInnen grundlegend zu verbessern.

Ziel des vorliegenden Beitrags ist es, die Ergebnisse der Befragung von Nutzerlnnen und NichtnutzerInnen für eine Produktentwicklung aufzubereiten und zu analysieren, inwiefern sich die Ergebnisse der Befragungen unterscheiden und welche Erkenntnisse die Befragung von NichtnutzerInnen generiert.

\section{Grundlagen der Nichtnutzung}

Dieses Kapitel befasst sich mit den unterschiedlichen Arten der Nichtnutzung, Gründe für eine Nichtnutzung und in welchen Formen diese in eine Produktentwicklung überführt werden können.

\section{Arten der Nichtnutzung}

Die Nichtnutzungskarte in Abbildung 1 visualisiert unterschiedliche Arten der Nichtnutzung, die auf Grundlage einer Literaturanalyse entwickelt wurde (Augustin, Kokoschko, Wiesner, Schabacker, 2020). Vertikal teilt sich die Karte in vier Kategorien der Nutzung und Nichtnutzung, von aktuell in Nutzung bis hin zu noch nie in Nutzung. Die Diagonale von links unten nach rechts oben beschreibt den Willen zur Nutzung, 
von starker Ablehnung (links unten) bis hin zur freiwilligen Nutzung (rechts oben). Diesen Kategorien sind die Definitionen aus der Literatur zugeordnet, um einen umfassenden Überblick über den Verlauf von Nutzung zu Nichtnutzung und zurück zu geben.

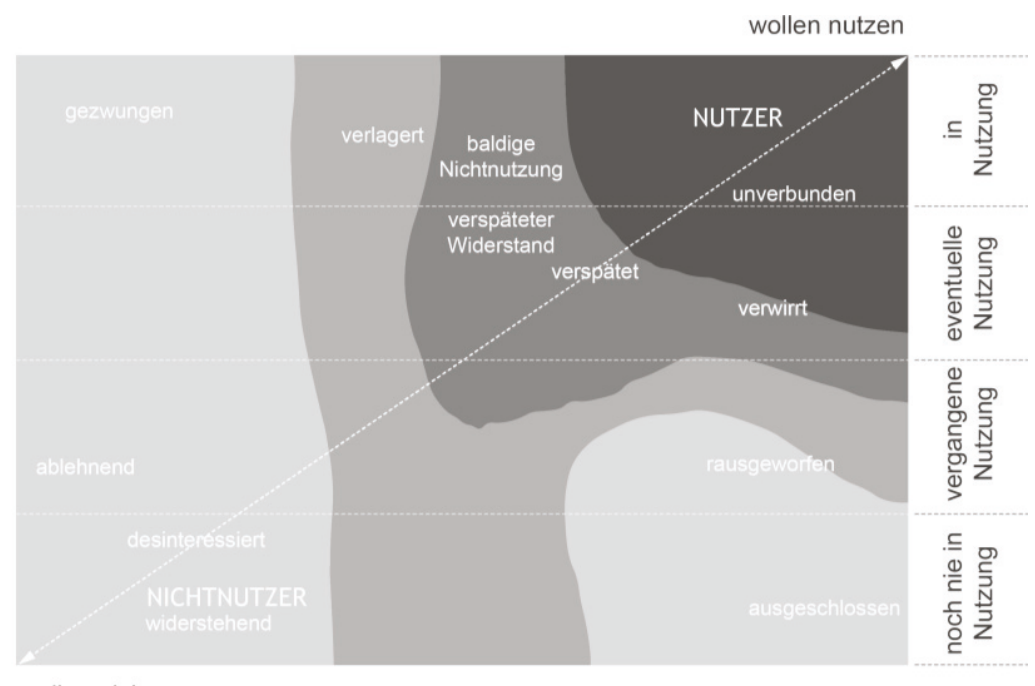

wollen nicht nutzen

Abbildung 1: Karte der Arten von Nichtnutzung (in Anlehnung an Augustin, Kokoschko, Wiesner, Schabacker, 2020)

Diese Karte kann unter anderem genutzt werden, um Ergebnisse aus Befragungen zu ordnen und den jeweiligen Kategorien zuzuordnen oder auch um im Vorhinein bestimme Gruppen zu identifizieren, die für ein Produkt gezielt untersucht werden sollen.

\section{Gründe für eine Nichtnutzung}

Die Gründe für eine Nichtnutzung sind in Abbildung 2 auf der Grundlage einer Literaturanalyse zusammengefasst (Augustin, Kokoschko, Wolffram, Schabacker, 2021) und teilen sich in drei Dimensionen ein: Person, Interaktion und Produkt (Ali, Zhou, Miller \& leromonachou, 2016; Jiang, Muhanna \& Klein, 2000). Beeinflusst werden diese von den drei Rahmen: persönlich, produktbezogen und sozial. Der individuelle Rahmen umfasst persönliche Daten wie z. B. Alter oder Geschlecht, den ökonomischen Status, Persönlichkeit, Entscheidungsfindung, aber auch Gewohnheiten, Überzeugungen und Routinen. Der soziale Rahmen beschreibt das Umfeld der Person, Familie, Freunde, Arbeit, Religion und Kultur. Der Markt- und Produktrahmen beschreibt produktspezifische Aspekte wie Qualität, Preis, Wechselkosten, Sicherheit und Instandhaltung sowie 
die Anzahl der auf dem Markt enthaltenen Produkte. Die Überschneidung aus Produkt und Person bildet die Interaktion mit den Unterpunkten Zugänglichkeit, Nutzungsfreundlichkeit, Gebrauchstauglichkeit, Verständlichkeit, Erwartungen und Markenbild.

Die Beeinflussbarkeit dieser Aspekte von Produktentwicklung ist sehr unterschiedlich, besonders da der individuelle und soziale Rahmen mit einem Produkt schwer erreichbar ist. Trotzdem sollten diese bei einer Entwicklung zumindest als Einflussfaktoren bekannt sein.

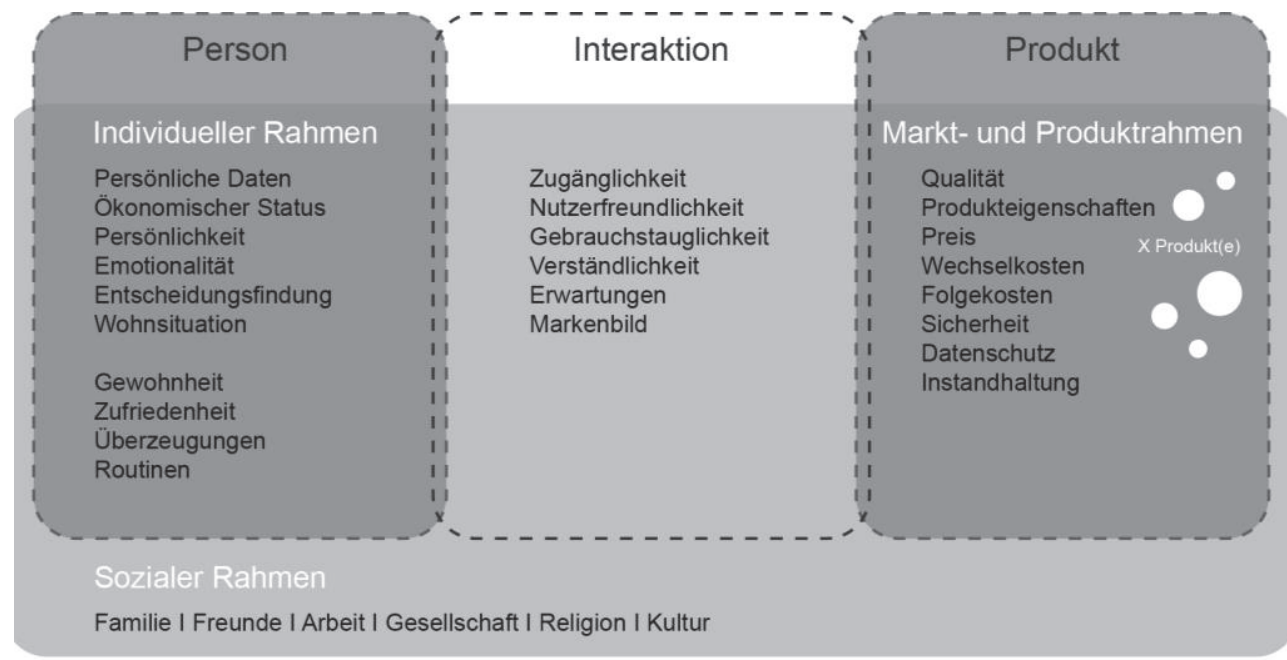

Abbildung 2: Gründe für eine Nichtnutzung

Neben den unterschiedlichen Einflussfaktoren lassen sich in der Literatur auch noch weitere Aspekte zum Thema Gründe für eine Nichtnutzung finden, so beispielsweise auch Barrieren wie Nutzungsbarriere (Gewohnheit), Wertbarriere (Wertsteigerung für Nutzende nicht erkennbar), Risikobarriere (physische, funktionale und soziale Risiken) und Traditionsbarriere (Konflikt zu Normen des Nutzenden) (Gurtner, 2014). Diese Barrieren sind als Gründe für eine Nichtnutzung in Abbildung 2 miteinbezogen.

\section{Integration von Nichtnutzung in die Produktentwicklung mithilfe der Non-Persona}

Die Non-Persona (Augustin, Kokoschko, Wolffram, Schabacker, 2021) beschreibt das Gegenüber zur Persona (A. Cooper, 2004), um Eigenschaften und Ansichten der NichtnutzerInnen zu visualisieren. Im Gegensatz zur affected (=betroffenen) persona (Karwowski, 2011), die nur von der Nutzung betroffene Personen beschreibt, umfasst 
die Non-Persona alle NichtnutzerInnen. Die negative Persona oder Antipersona (A. Cooper, Noessel, Cronin \& Reimann, 2014) beschreibt Personen, für die das Produkt nicht entwickelt wird oder für die das Produkt nicht gedacht ist (Brangier \& Bornet, 2011). Sie kann auch als abschreckendes Beispiel dienen, das man für eine Entwicklung im Hinterkopf behalten sollte (Wobig, 2012). Mithilfe der Non-Persona sollen jedoch Personengruppen visualisiert werden, die in eine Produktentwicklung miteinbezogen werden sollen, um zum Beispiel den Markt zu erweitern.

Einige Aspekte sind für Persona und Non-Persona relevant, wie demographische Daten, Persönlichkeit, Interessen, Hobbys, Technikvorlieben und Tagesablauf sowie Produktfeedback (Nielsen, Hansen, Stage, Billestrup, 2015). Die beiden in den Abschnitten 2.1 und 2.2 diskutierten Aspekte Nichtnutzung und Gründe für eine Nichtnutzung werden noch zusätzlich in die Non-Persona, visualisiert in Abbildung 3, eingebunden.
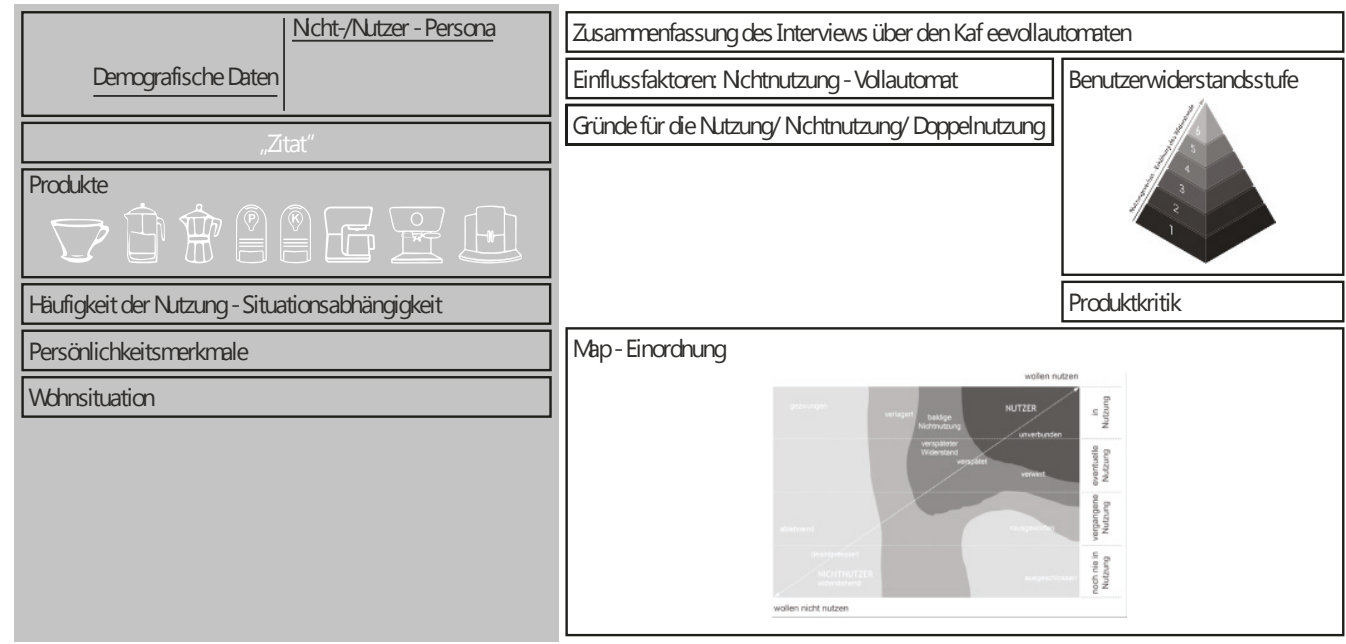

Map-Einordnung

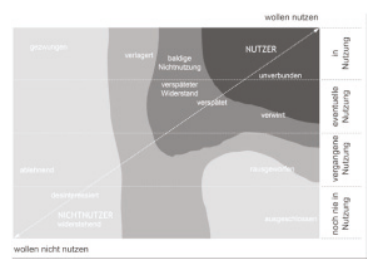

Abbildung 3: Struktur Non-Persona

\section{Fallstudie: Entwicklung eines Kaffeeautomaten}

Dieses Kapitel befasst sich mit einer Fallstudie, in der die Befragung von NichtnutzerInnen und NutzerInnen durchgeführt und analysiert wurde. Zunächst wird auf die Struktur der Fallstudie eingegangen, im darauf folgenden Abschnitt die Ergebnisse vorgestellt und abschließend mit KooperationspartnerInnen aus der Industrie diskutiert und für die weitere Arbeit mit Nichtnutzung eingeordnet. 


\section{Aufbau und Ablauf der Befragung}

Die Befragung fand als persönliche Interviews (33 Personen) und Onlinebefragung (42 Personen) statt, insgesamt wurden also 75 Personen befragt. Davon sind 20 Personen NutzerInnen eines Kaffeeautomats und 55 Personen NichtnutzerInnen. In Abbildung 4 sind alle Befragten nach Alter, Geschlecht und Nutzungsart dargestellt. Die Befragung (persönlich und online) beginnt mit einer kurzen Vorstellung des Interviewablaufs und umfasst die Themen Produktbesitz, Nutzung, Produkterfahrungen, Produkterhalt, Gewohnheiten zum Kaffeekonsum, Wohnsituation, Kaffeetrinksituationen und Betreuungsaufwand seitens der (Nicht-)NutzerInnen. Zuletzt wird eine Einordnung in die Karte der Nichtnutzung und Gründe für eine Nichtnutzung vorgenommen. Die in der Befragung behandelten Produkte sind in Abbildung 5 dargestellt. Im Bereich der Nichtnutzung von Kaffeevollautomaten werden die herkömmlichsten Herstellungswege für Kaffee abgefragt, eingeteilt in händisch, mechanisch und maschinell. Die einzigen Produkte, die mit der Befragung nicht abgedeckt wurden, sind Aeropress, Bodum, Cold Brew und türkischer Kaffee.

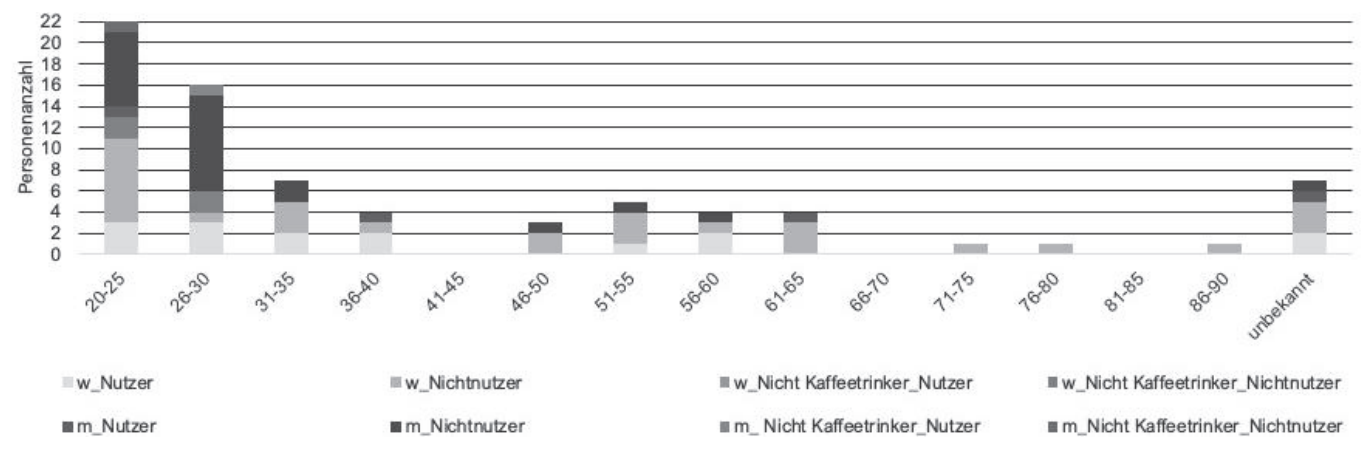

Abbildung 4: Übersicht Befragte mit Alter und Geschlecht

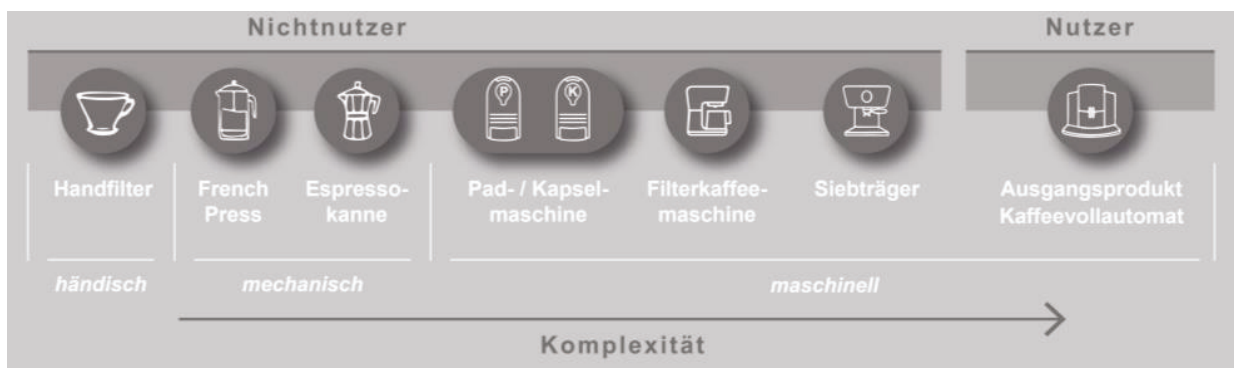

Abbildung 5: Produktauswahl 


\section{Ergebnisse der Befragung}

Die Ergebnisaufbereitung der Befragung teilt sich in allgemeine Beobachtungen zur Kaffeezubereitung, dem Entscheidungsprozess beim Kauf, die Gründe für eine Nichtnutzung und eine Einordnung aller Befragten auf der (Nicht)nutzungskarte. Zudem wird eine beispielhafte Non-Persona aus den Daten der NichtnutzerInnen erstellt.

Etwa 50 \% der befragten Personen nutzen mindestens zwei Produkte zur Kaffeezubereitung, zwölf Personen nutzen sogar drei Produkte und drei Personen nutzen vier. Es besteht also der Bedarf nach Produktkombinationen, da ein Produkt bei der Hälfte der Personen nicht alle gewünschten Eigenschaften umfasst.

Abbildung 6 visualisiert die Nutzungs- bzw. Nichtnutzungstypen der Befragten und wie sie sich zwischen den Dimensionen der Karte verorten lassen. Es lassen sich hierbei drei Cluster feststellen:

- die „Verspäteten“: diese Gruppe ist sich noch unsicher, ob sie das Produkt nutzen möchten, stehen aber kurz vor der Entscheidung.

- Ein Cluster nahe der „Verspäteten“, sie stehen ebenfalls kurz vor einer Nutzung, lehnen das Produkt jedoch etwas mehr ab (und sind daher weiter links auf der Karte verortet).

— die Nichtnutzenden: ablehnend, desinteressiert und widerstehend. Diese Gruppe lehnt das Produkt kategorisch ab.

In Abbildung 7 wird das Thema Produktwanderung visualisiert, also eine Art Historie der Produktnutzung. Es wird dargestellt, von welchen Produkten die Befragten hin zum Kaffeeautomat wechseln und wohin bisherige Nutzerlnnen abgesprungen sind. Hierbei lässt sich feststellen, dass die häufigste Produktwanderung weg vom Kaffeevollautomaten hin zur Siebträgermaschine ging. Zum Kaffeevollautomaten wechselten hauptsächlich NutzerInnen von Espressokannen und Filtermaschinen.

Abbildung 8 zeigt eine Non-Persona angelehnt an das vorgestellte Konzept aus Abschnitt 2.3, welche auf den Ergebnissen der Befragungen beruht. Die vorliegende NonPersona beschreibt ein verheiratetes Paar zwischen 30 und 35, und ein kurzes Zitat gibt einen ersten Überblick über den Charakter der beiden. Sie nutzen eine French Press und eine Siebträgermaschine, letztere benutzen sie hauptsächlich, wenn es um den Geschmack geht. Die French Press wird genutzt wenn es um Menge oder Schnelligkeit geht. Darunter finden sich Persönlichkeitsmerkmale, die sich aus Persönlichkeit, Hobbys, Lebensstil und Produktzufriedenheit zusammensetzen. Die linke Spalte endet 
mit einem Überblick über die Wohnsituation, die die Punkte Wohnungsaufbau, Haushaltsgröße, Besuch und Kaffeekonsum umfasst.

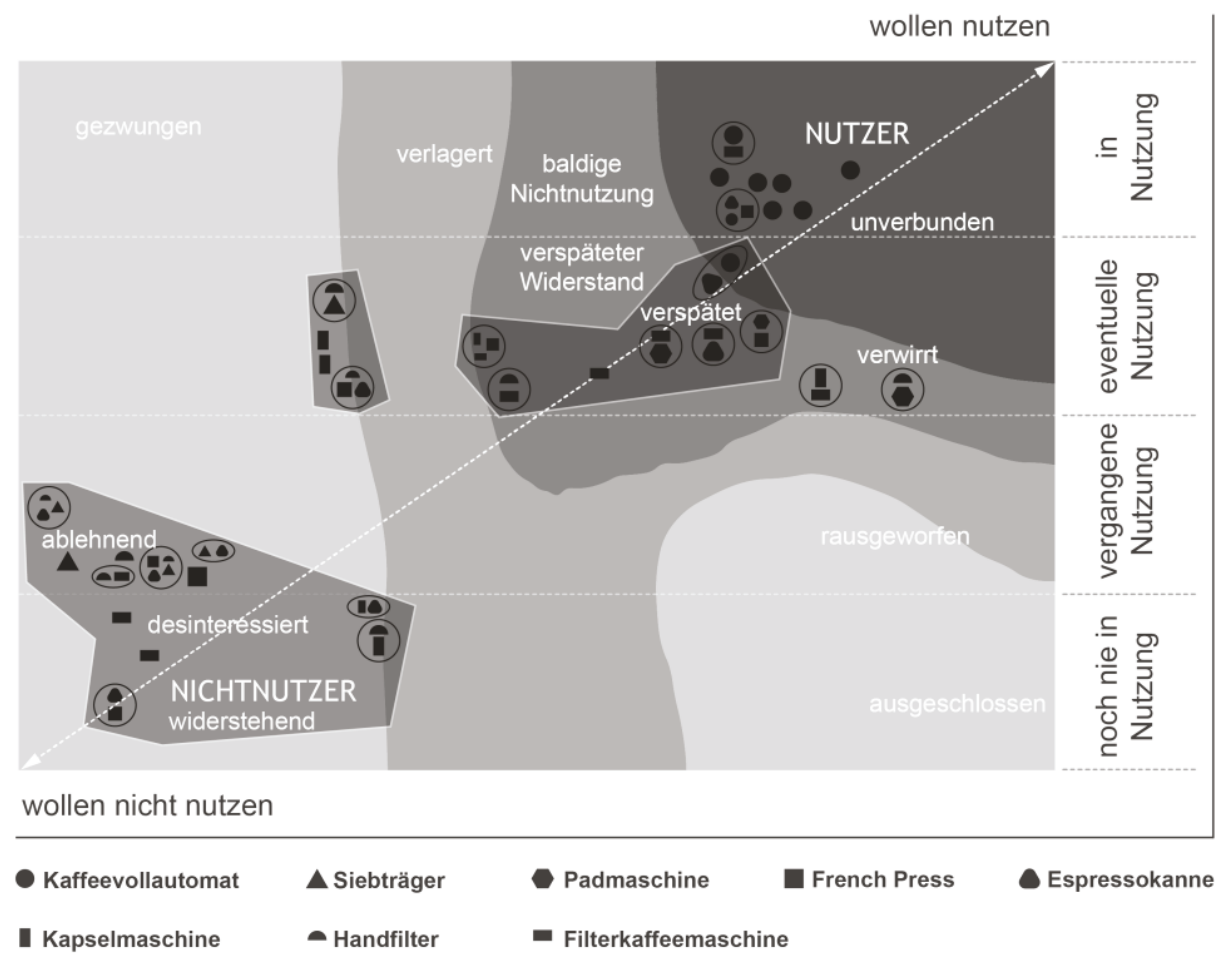

Abbildung 6: Nichtnutzungskarte Kaffeeautomat

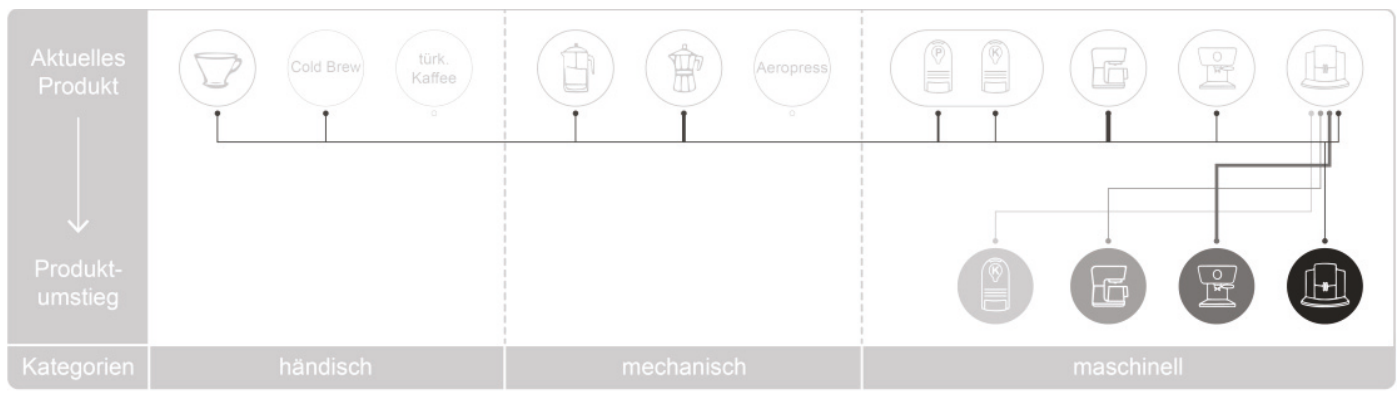

Abbildung 7: Überblick Produktwanderung 


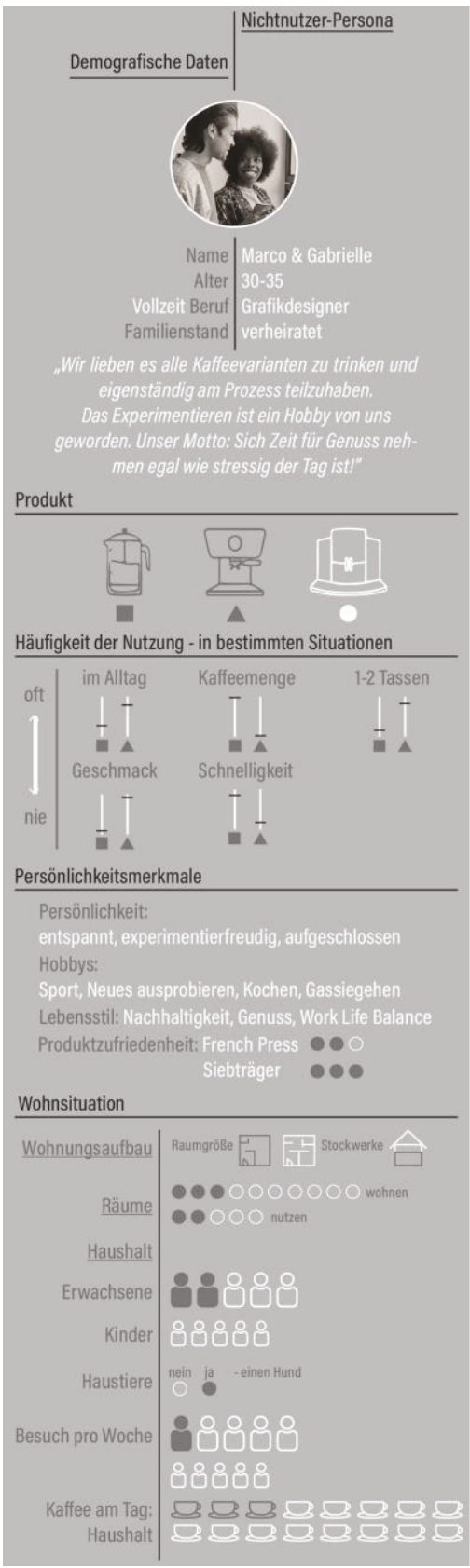

Zusammenfassung des Interviews über den Kaffeevollautomaten

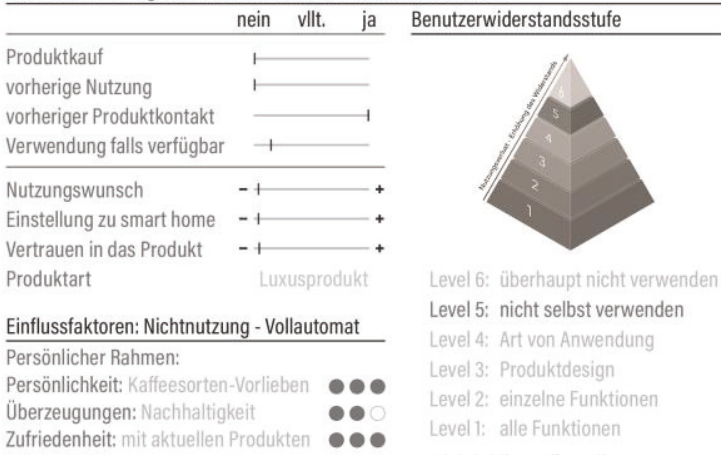

\section{Interaktion:}

Nutzerfreundlichkeit: Überforderungen

Erwartungen: Geschmack nicht gut

Markenbild: Massenabfertigung

Markt- und Produktrahmen:

Instandhaltung: Reinigungsaufwand

Preis: zu teuer, kann nichts richtig

Produkteigenschaften: veraltet

Gründe für die Nutzung/ Nichtnutzung/ Doppelnutzung

- keine Entschleunigung im Alltag möglich, Automatismus - keine Möglichkeit an

Zubereitungsprozess teilhaben zu könner

- negatives Produktbild: das Innere des Kaffeevollautomaten verschimmelt

- viele Familienmitglieder nutzen einen Siebträger - Kaufempfehlung

- Kaffeevollautomaten vs. Siebträger im Geschäft: Geschmack des Siebträgers

hat überzeugt

- Reparaturen Iohnen sich beim Kaffeevollautomaten nicht \& die Produktfunktionen veralten - nicht nachhaltig

- keine zufriedenstellende \& e eigenständige Maschinenreinigung möglich - nicht sichtbar

Karten - Einordnung

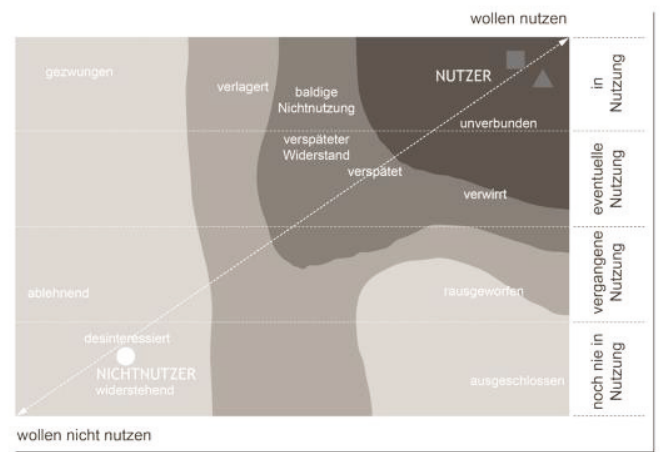

\section{Abbildung 8: Non-Persona Kaffeevollautomat}


Die rechte Seite der Non-Persona beschreibt neben der Ablehnungsintensität auf der Pyramide noch die Einstellung zum nichtgenutzten Kaffeevollautomaten. In diesem Fall ist der Widerstand auf Level 5, also sehr hoch, und der Nutzungswunsch sehr niedrig. Die Einflussfaktoren auf die Nichtnutzung sind im Fall von Marco und Gabrielle eigene Vorlieben, die Zufriedenheit mit den aktuellen Produkten, geringe Erwartungen an den Kaffeevollautomaten und der befürchtete Reinigungsaufwand. Neben der Produktkritik am Kaffeevollautomat werden noch Gründe für die Nichtnutzung aufgeführt und eine Einordnung in die Nichtnutzungskarte getätigt. Die beiden fallen in den Bereich desinteressiert/widerstehend am unteren Ende links, den Bereich mit dem größten Widerstand.

Die Non-Persona kann ergänzend zu Personas im Entwicklungsalltag eingesetzt werden, um so eine weitere Perspektive in den Prozess einzubringen.

\section{Feedback aus der Industrie}

In einer Diskussionsrunde mit KooperationspartnerInnen aus der Industrie wurden die Ergebnisse der Befragungen vorgestellt. Die Gründe für eine Nichtnutzung ballt sich im Bereich der Interaktion in den Aspekten Gebrauchstauglichkeit, Verständlichkeit und Erwartungen was in der Diskussion auf eventuell fehlendes Produktwissen zurückgeführt wird und somit wertvollen Input für das Marketing liefert. Die Beobachtung, dass der Umstieg weg vom Kaffeevollautomaten meist hin zur Siebträgermaschine geht, offenbart diese als einen relevanten und bisher wenig betrachteten Mitbewerber. Die Ergebnisse hinsichtlich der Anforderungen von NichtnutzerInnen deckt sich jedoch mit den Ergebnissen der unternehmenseigenen Befragung von Nutzerlnnen.

\section{Diskussion und Einordnung der Ergebnisse}

Dieser Beitrag beschreibt auf Grundlage einer Fallstudie das Thema Nichtnutzung im Kontext des Kaffeeautomaten. Die Fallstudie dient der Konkretisierung der Ergebnisse aus der vorangegangenen Literaturanalyse. Da sich die Studie auf ein spezifisches Produkt bezieht, ist die Reichweite der Ergebnisse dementsprechend begrenzt und können für weitere Produkte nicht automatisch angewendet werden. Weitere Untersuchungen sind nötig, um eine Aussage über die Allgemeingültigkeit der Ergebnisse treffen zu können. Da es sich bei dem Produkt Kaffeevollautomat um ein am Markt etabliertes Produkt handelt, sind hierbei die (Nicht-)NutzerInnen weitestgehend bekannt. Im Rahmen einer neuen Produktkategorie, bei der diese möglicherweise nicht bekannt sind, können Annäherungen beispielsweise über die Non-Persona (Augustin, 
Kokoschko, Wolffram, Schabacker, 2021) gemacht werden, um potentielle Befragungsgruppen zu finden. Die Karte der Nichtnutzung kann zudem als Einordnung oder Eingrenzung genutzt werden, bevor erste Befragungen durchgeführt werden.

\section{Zusammenfassung}

Auf Grundlage vorhergehender Literaturanalysen (Augustin, Kokoschko, Wiesner, Schabacker, 2020; Augustin, Kokoschko, Wolffram, Schabacker, 2021) werden in diesem Beitrag Nutzung und Nichtnutzung von Kaffeeautomaten analysiert und verglichen. Auf dieser Grundlage werden unterschiedliche Visualisierungen und Einordnungen der Ergebnisse aus den Umfragen getätigt (wie z. B. die Non-Persona und die Nichtnutzungskarte) und mit EntwicklerInnen aus der Industrie diskutiert und eingeordnet. Weitere quantitative Studien sind erforderlich, um die gewonnenen Erkenntnisse der qualitativen Untersuchung zu verifizieren und bei Bedarf zu erweitern. Dieser Beitrag stellt heraus, welches Potential die Einbindung der Nichtnutzung in eine Produktentwicklung hat und zeigt mithilfe der Karte der Nichtnutzung und Non-Persona auf, wie Befragungen von (Nicht-)NutzerInnen in anwendbare Visualisierungen übersetzt werden können.

\section{Literaturverzeichnis}

Ali, M., Zhou, L., Miller, L. \& leromonachou, P. (2016). User resistance in IT: A literature review. International Journal of Information Management, 36(1), 35-43. https://doi.org/10.1016/j.jijnfomgt.2015.09.007

Augustin, L., Kokoschko, B., Wiesner, M. \& Schabacker, M. (2020). Toward a comprehensive definition of the non-user (Proceedings of the Design Society: DESIGN Conference, Bd. 1). DOl: https://doi.org/10.1017/dsd.2020.124

Augustin, L., Kokoschko, B., Wolffram, A. \& Schabacker, M. (2021). Defining the non-user: a classification of reasons for non-use (Proceedings of the $8^{\text {th }}$ International Conference on Research into Design, Icord), in Druck

Barsch, T., Heupel, T. \& Trautmann, H. (Hrsg.). (2019). Die Blue-Ocean-Strategie in Theorie und Praxis. Wiesbaden: Springer Fachmedien Wiesbaden. https://doi.org/10.1007/978-3-658-15480-6

Brangier, E. \& Bornet, C. (2011). Persona: A Method to Produce Representations Focused on Consumers' Needs. In W. Karwowski (Ed.), Human factors and ergonomics in consumer product design. Methods and techniques (Ergonomics design and management, / ed. by Waldemar Karwowski ... ; Vol. 1, S. 37-62). Boca Raton, Fla.: CRC Press.

Brenner, W., Uebernickel, F. \& Abrell, T. (2016). Design Thinking as Mindset, Process, and Toolbox (Design Thinking for Innovation): Springer International Publishing. Verfügbar unter: http://dx.doi.org/10.1007/978-3-319-26100-3_1

Cooper, A. (2004). The Inmates Are Running the Asylum. Why High-Tech Products Drive Us Crazy and How to Restore the Sanity ( $2^{\text {nd }}$ edition). Sams. 
Cooper, A., Noessel, C., Cronin, D. \& Reimann, R. (2014). About face. The essentials of interaction design (4 ${ }^{\text {th }}$ edition). Indianapolis, IN: Wiley. Retrieved from http://gbv.eblib.com/patron/FullRecord.aspx?p=4038509

Cooper, R. G. \& Kleinschmidt, E. J. (1986). An Investigation into the New Product Process: Steps, Deficiencies, and Impact. Journal of Product Innovation Management, 3(2), 71-85. https://doi.org/10.1111/1540-5885.320071

Doorley, S., Holcomb, S., Klebahn, P., Segovia, K. \& Utley, J. (2018). Design Thinking Bootleg, d.school at Stanford University. Zugriff am 08.03.2021. Verfügbar unter: https://drive.google.com/file/d/1qfrW8qu0lbl2vfxqHSN7VH03ACzpHJ4a/view

Gardan, J. (2017). Definition of users' requirements in the customized product design through a user-centered translation method. International Journal on Interactive Design and Manufacturing (IIIDeM), 11(4), 813-821.

https://doi.org/10.1007/s12008-015-0275-2

Gurtner, S. (2014). Modelling consumer resistance to mobile health applications. In M. Avital, J. M. Leimeister \& U. Schultze (Hrsg.), Proceedings of the European Conference on Information Systems (ECIS) (June 9-11, ISBN 978-0-9915567-0-0). Tel Aviv. Verfügbar unter: https://aisel.aisnet.org/cgi/viewcontent.cgi?article=1007\&context=ecis2014

Hippel, E. von. (1976). The dominant role of users in the scientific instrument innovation process. Research Policy, 5(3), 212-239. https://doi.org/10.1016/0048-7333(76)90028-7

Ind, N. \& Coates, N. (2013). The meanings of co-creation. European Business Review, 25(1), 86-95. https://doi.org/10.1108/09555341311287754

Jiang, J. J., Muhanna, W. A. \& Klein, G. (2000). User resistance and strategies for promoting acceptance across system types. Information \& Management, 37(1), 25-36. https://doi.org/10.1016/50378-7206(99)00032-4

Karwowski, W. (Ed.). (2011). Human factors and ergonomics in consumer product design. Methods and techniques (Ergonomics design and management, / ed. by Waldemar Karwowski ... ; Vol. 1). Boca Raton, Fla.: CRC Press.

Kurosu, M. (2011). Human Centered Design (Bd. 6776). Berlin, Heidelberg: Springer Berlin Heidelberg. https://doi.org/10.1007/978-3-642-21753-1

Lemon, K. N. \& Verhoef, P. C. (2016). Understanding Customer Experience Throughout the Customer Journey. Journal of Marketing, 80(6), 69-96. https://doi.org/10.1509/jm.15.0420

Murphy, S. A. \& Kumar, V. (1997). The front end of new product development: a Canadian survey. R and D Management, 27(1), 5-15. https://doi.org/10.1111/1467-9310.00038

Nielsen, L., Hansen, K. S., Stage, J. \& Billestrup, J. (2015). A Template for Design Personas. International Journal of Sociotechnology and Knowledge Development, 7(1), 45-61. https://doi.org/10.4018/ijskd.2015010104

Ribak, R. \& Rosenthal, M. (2015). Smartphone resistance as media ambivalence. First Monday, 20(11). https://doi.org/10.5210/fm.v20i11.6307

Satchell, C. \& Dourish, P. (2009). Beyond the user: Use and non-use in HCl. In M. Foth (Hrsg.), Proceedings of the 21st Annual Conference of the Australian Computer-Human Interaction Special Interest Group (CHISIG) of the Human Factors and Ergonomics Society of Australia (HFESA), (S. 9). New York: ACM.

Sutherland, J. \& Schwaber, K. (1993). Scrum Guide. Zugriff am 08.03.2021. Verfügbar unter: https://scrumguides.org/scrumguide.html 
Wobig, M.. Benutzermodellierung mit Personas und Zielen, Leibniz Universität Hannover. Zugriff am 08.03.2021. Verfügbar unter: http://www.se.uni-hannover.de/pub/File/kurz-und-gut/ss2012-proseminar-inf-usabiliy/Wobig2012.pdf

Wyatt, S. (2003). Non-users also matter: The construction of users and non-users of the Internet. In N. Oudshoorn \& T. J. Pinch (Eds.), How users matter. The co-construction of users and technologies (Inside technology, S. 67-80). Cambridge, Mass: MIT Press.

\section{Kontakt}

Laura Augustin, M. Sc.

Sabrina Pfrang, B. A.

Björn Kokoschko, M. A.

Gast-Prof. Dr.-phil. Andrea Wolffram

Dr.-Ing. Dipl.-Math. Michael Schabacker

Otto-von-Guericke-Universität Magdeburg

Fakultät für Maschinenbau

Institut für Maschinenkonstruktion

Lehrstuhl Produktentwicklung und Konstruktion

Universitätsplatz 2

39106 Magdeburg

Ipk.ovgu.de 